\title{
QUALIDADE DE VIDA E AUTISMO
}

\author{
Alexsandra V. Elias', Francisco B. Assumpção $\mathrm{Jr}^{2}$
}

RESUMO - Objetivo: Avaliar a qualidade de vida (QV) de crianças com transtornos invasivos do desenvolvimento. Método: 20 meninos autistas, entre 4 e 12 anos, submetidos à escala de traços autísticos-ATA e a Vineland adaptive behavior scales, necessitando obter nesta, nível de desenvolvimento adaptativo igual ou maior que 70. Os dados sobre a QV foram obtidos pela Escala de Qualidade de Vida - AUQEI e comparados a partir da aplicação da Vineland e da AUQEI em 20 crianças normais, pareadas quanto a sexo e idade. Resultados: Os índices da Vineland sugerem que crianças normais apresentam melhor desempenho adaptativo do que crianças autistas $(p=0,0196)$. Nos dados sobre $Q V$, verificou-se que os índices gerais são iguais para ambos os grupos, indicando QV positiva $(\mathrm{p}=0,744)$, entretanto; no subdomínio autonomia, crianças autistas apresentaram índices mais elevados $(p=0,0048)$. Conclusão: Crianças autistas apresentam índices QV iguais aos índices de crianças normais.

PALAVRAS-CHAVE: qualidade de vida, infância, autismo.

\section{Quality of life and autism}

ABSTRACT - Objective: To evaluate the index quality of life (QL) in bearers of including upset of the development. Method: 20 autistic childrens, between 4 and 12 years, submitted to the diagnostic evaluation by autistic traces scale-ATA and for the Vineland adaptive behavior scales, needing to obtain in this a quotient of superior development over 70. The data about QL were obtained by the scale of quality of lifeAUQEI and compared from the application of Vineland and of AUQEI in a population of normal children, resembling in the sex and in the age. Results: In the evaluation of the ATA, average was 33.75 with 4.95 standarddeviation. The indexes of Vineland suggest that normal children have larger probability to present level of appropriated adaptative behavior than autistic children $(p=0.0196)$. Concerning $Q L$, the general indexes are the same for both groups, indicating positive $Q L(p=0.744)$. In the sub domain autonomy, autistic children present higher index $(p=0.0048)$. Conclusion: Autistic childrens present similar indexes of QL than normal childrens.

KEY WORDS: quality of life, childhood, autism.

O autismo está classificado na subcategoria dos transtornos invasivos do desenvolvimento e inclui prejuízos na interação social, na comunicação, padrões restritos e repetitivos de comportamento, interesses, atividades e início antes dos 3 anos de idade'1. As manifestações clínicas variam amplamente em termos de níveis de gravidade, algumas crianças, conhecidas como "autistas de alto funcionamento", podem chegar a se desenvolver de maneira parcial e relativamente independente e apresentar nível intelectual dentro da normalidade 2 , o que lhes permitem condições de manifestarem-se em relação às suas percepções de qualidade de vida (QV). QV é um conceito subjetivo que inclui a percepção do indivíduo em sua posição na vida, no contexto cultural e no sistema de valores em que vive e em relação a seus objetivos, expectativas, padrões e preocupações ${ }^{3}$.

O estudo da QV em crianças autistas pode proporcionar um meio de compreender possibilidades dessa população. Garante-lhes um olhar pessoal, não só em termos das condições inerentes ao quadro, mas possibilitando o planejamento de intervenções que visem contribuir ou adequar-se à visão da criança baseandose em suas condições específicas. Partimos da premissa que, quando portadora de melhores níveis de desenvolvimento, essas crianças poderão se adaptar ao ambiente e desenvolver melhor índice de QV, entendida aqui, quanto ao bem-estar referente ao grau de satisfação em vários domínios das suas vidas ${ }^{4,5}$.

O objetivo deste estudo é avaliar o índice de QV

${ }^{1}$ Psicóloga, Mestre em Ciências Médicas pela Universidade Estadual de Campinas (FCM-UMICAMP), Campinas SP, Brasil; ${ }^{2}$ Psiquiatra, P rofessor Livre Docente do Departamento de Psiquiatria da Faculdade de Medicina da Universidade de São Paulo (FMUSP), São Paulo SP, Brasil.

Recebido 7 Junho 2005, recebido na forma final 3 Novembro 2005. Aceito 23 Janeiro 2006.

Dra. Alexsandra Vieira Elias - Departamento de Psicologia Médica e Psiquiatria/FCMIUnicamp / Caixa Postal 6111 -13081-970 Campinas SP - Brasil. E-mail: alexsandrabender@hotmail.com 
de crianças autistas e verificar se suas percepções diferem ou não das percepções de crianças normais, de mesma idade, sexo e grupo social.

\section{MÉTODO}

Este estudo foi desenvolvido após ter sido aprovado pelo Comitê de Ética em Pesquisa da FCM/UNICAMP, com acompanhamento de amostra de 40 crianças. A coleta de dados foi precedida de informações sobre os objetivos e procedimentos da pesquisa junto às mães das crianças, as quais eram solicitadas a assinar o termo de consentimento pós-informado em que autorizavam a participação da criança no estudo.

O grupo experimental contou com coleta de conveniência; as crianças foram localizadas de forma aleatória em várias cidades na região de Campinas, nas cidades de São Paulo e Ribeirão Preto, subordinando-se a raridade do perfil estabelecido pela pesquisa.

A coleta no grupo controle foi realizada em dezembro a janeiro de 2004, em um bairro de classe média alta na cidade de Alfenas - MG, por contato direto com os responsá veis pelas crianças e com estas sem a mediação de instituições da cidade, visto que se tratava de período de férias escolares.

Foram estudados dois grupos, constituídos da seguinte forma:

G rupo A - 20 crianças autistas do sexo masculino, entre 4 e 12 anos. Tais crianças já tinham um diagnóstico clínico segundo os critérios do DSM- IV, porém, foram avaliadas pela escala de traços autísticos-ATA ${ }^{6}$, traduzida e validada no Brasil7, para maior confiabilidade diagnóstica. Também foram submetidas à "Vineland adaptative behavior scale" para que fossem incluídas na amostra somente crianças cujo índice de comportamento adaptativo tivesse pontuação igual e/ou acima de 70. Isto porque a pesquisa teria de envolver crianças cujo perfil fosse compatível ao grupo controle.

Tal escala é usada em muitos estudos para avaliar níveis de comportamento adaptativo e habilidades sociais, em autistas e outras inabilidades do desenvolvimento. É composta de vários subdomínios: domínio comunicacional, atividades da vida cotidiana, domínio social, habilidades motoras, comportamento disruptivo. Esses dois últimos subdomínios não foram incluídos neste estudo, devido à faixa etária estabelecida.

$G$ rupo $B-20$ crianças normais, pareadas quanto a sexo e idade com o grupo anterior, avaliadas pela "Vineland adaptative behavior scale" 8 .

Posteriormente, ambos os grupos foram submetidos à escala de QV AUQEI ${ }^{4}$, traduzida e validada em nosso meio ${ }^{5}$. Trata-se de instrumento genérico, aplicável em crianças de 4 a 12 anos, que se baseia na perspectiva subjetiva da criança, apresentada em quatro fatores distintos: funções, família, lazer e autonomia ${ }^{4,5,9}$. Seu ponto de corte é 48 , ou seja, valores iguais ou acima de 48 indicavam QV satisfatória ${ }^{5}$.

\section{RESULTADOS}

O ponto de partida foi estabelecer a média geral da pontuação obtida pela escala de traços autísticos - ATA. A partir destes dados, foi calculado o desviopadrão, com nota de corte de (15), ou seja, valores iguais ou maiores que 15 seriam considerados autistas $^{7}$, obtendo um valor médio de 33,75 com desvio padrão de 4,95. O objetivo dessa etapa foi confirmar o diagnóstico clínico.

Seguiu-se análise preliminar da escala de Vineland, usando a análise exploratória e descritiva dos dados de forma que fosse possível identificar tendências, similaridades e diferenças entre os grupos. Foram construídas tabelas de contingência (freqüência e porcentagem), com o objetivo de se realizar testes que verificassem igualdade ou diferença entre os grupos de crianças.

Foi utilizado o teste binominal, com intuito de verificar se as crianças dos dois grupos possuíam nível da escala de Vineland (e dos subdomínios) similar ou diferente. A escolha de tal teste foi feita com base no tamanho da amostra e nas características dos dados coletados, uma vez que em todos os índices havia níveis que não tinham sido observados para um dos g rupos, ou apresentavam freqüência pequena (algumas menores que 5 e outras iguais a zero) impossibilitando o uso de testes não exatos.

O índice da Vineland no domínio comunicacional não apresentou diferenças significantes entres os grupos de acordo com os níveis $(p=0,8067)$ para o nível moderado baixo e adequado $(p=0,2844)$. Porém, os índices da Vineland na atividade de vida cotidiana apresentaram diferença significante entre crianças autistas e não autistas nos níveis moderado baixo e adequado, através do teste binomial realizado. Verificou-se, que a probabilidade de uma criança autista, dessa amostra, ter atividade de vida cotidiana moderada baixa é maior que uma criança não autista ( $p=$ 0,0013 ) e a probabilidade de uma criança, dessa amostra, não autista ter atividade de vida cotidiana adequada é maior de que uma criança autista ter esse nível $(p=0,0104)$.

O domínio social também apresentou diferença significativa nos níveis moderado baixo e adequado ao se realizar o teste exato. Foi possível verificar maior p robabilidade de uma criança autista apresentar domínio social moderado baixo do que uma criança não autista ( $p=0,0064)$, e maior probabilidade de uma criança não autista ter domínio social adequado do que uma criança autista $(p=0,0409)$. Da mesma maneira, detectou-se que há diferença entre os grupos para os níveis moderado baixo e adequado no escore total da Vineland (Tabela 1). 
Conformea T1, observamos que a probabilidade de crianças autistas apresentarem índice de comportamento adaptativo moderado baixo para essa amostra é maior do que crianças não autistas apresentarem esse nível $(p=0,0029)$, e crianças não autistas têm maior p robabilidade de apresentarem nível de comportamento adaptativo adequado do que crianças autistas $(p=0,0196)$.

Nos dados sobre QV, utilizou-se inicialmente o teste $t$ de comparação de médias, cujo objetivo foi comparar a pontuação média de um grupo com o outro, ou seja, verificar se há diferença entre a média da pontuação da AUQEI para crianças autistas e não autistas.

Com $95 \%$ de significância, o teste t não rejeitou a hipótese inicial de que as médias da pontuação AUQEI eram as mesmas para os dois grupos $(p=0,910)$. $A$ variável AUQEI também foi testada em forma de dois grupos (Tabela 2), um de crianças com AUQEI abaixo de 48 caracterizando QV prejudicada e outro de crianças com AUQEI acima ou igual a 48.

Obsenamos pela Tabela 2 que crianças autistas e não autistas apresentam mesma média de pontuação AUQEI. Realizando o teste de qui-quadrado de Pearson confirma-se através da não rejeição da hipótese nula de que as médias são iguais, não havendo diferença entre a média da pontuação de AUQEI de crian- ças autistas e não autistas $(p=0,744)$. Verificou-se que os índices gerais de QV são iguais para ambos os grupos, indicando QV positiva.

Para avaliarmos melhor os resultados de QV, analisamos a relação entre os grupos nos escores parc i a is da AUQEI (funções, família, lazer e autonomia). Para cada grupo também foi feito o teste $t$ de comparação de médias, levando em consideração a pontuação nas questões referentes a cada grupo.

$O$ grupo de questões referentes às funções ( $p=$ $0,321)$, família $(p=0,385)$ e lazer $(p=0,826)$ não apresentou diferenças estatisticamente significativas para crianças autistas em relação às não autistas; entretanto, a pontuação média para repostas que caracterizam autonomia para crianças autistas difere estatisticamente da média das crianças não autistas $(p=$ 0,0048).

\section{DISCUSSÃO}

Obtivemos com a análise da ATA um valor médio de 33,75 com desvio padrão de 4,95. Assim, o valor médio de 33,75 obtido neste estudo, permitiu estabelecer um diagnóstico bastante confiável do quadro em questão, conforme sugerido no estudo de validação ${ }^{7}$, o que confirmou o diagnóstico clínico. Para meIhor adequação do estudo, fizemos uso da escala de Vneland ${ }^{8}$. Esta, além de nos ajudar a selecionar as

Tabela 1. Tabela cruzada de Vineland (escore total) por Grupo.

\begin{tabular}{lcccc}
\hline Freqüência & Porcentagem & \multicolumn{3}{c}{ Grupo } \\
\cline { 3 - 5 } & & Autistas & Não Autistas & Total \\
\hline Vineland & Moderado & 12 & 1 & 13 \\
& Baixo & $30,0 \%$ & $2,5 \%$ & $32,5 \%$ \\
& Adequado & 8 & 18 & 26 \\
& Moderado & $0,0 \%$ & $45,0 \%$ & $65,0 \%$ \\
Alto & $0 \%$ & 1 & 1 \\
& Total & 20 & $2,5 \%$ & $2,5 \%$ \\
& & 20 & 40 \\
& & & & $100 \%$ \\
\hline
\end{tabular}

Tabela 2. Tabela Cruzada de AUQEI por Grupo.

\begin{tabular}{lcccc}
\hline Freqüência & & \multicolumn{3}{c}{ Grupo } \\
\cline { 3 - 5 } & & Autistas & Não Autistas & Total \\
\hline AUQUEI & $<48$ & 7 & 8 & 15 \\
& $\geq 48$ & 13 & 12 & 25 \\
& Total & 20 & 20 & 40 \\
\hline
\end{tabular}


crianças autistas a serem incluídas na pesquisa, proporcionou a compreensão de como a síndrome autística afeta a vida diária dessas crianças e ofereceu suporte para estabelecermos o nível de comportamento adaptativo delas, ou seja, o nível de desenvolvimento e uso das habilidades necessárias para a realização da independência pessoal e suficiência social ${ }^{10}$.

Considerando que utilizamos uma amostra de crianças autistas de alto funcionamento comparadas com crianças normais, podemos observar que o índice da Vineland no domínio comunicacional não apresentou diferenças significantes, porém, isso não exclui o prejuízo semântico inerente à criança autista. Por outro lado, as habilidades verbais presentes nessas crianças (alto funcionamento) são pre dito res de meIhores níveis de comportamento adaptável ${ }^{10,11}$.

Quando comparadas aos índices da Vineland na atividade de vida cotidiana, os resultados sugeriram diferença significante entre crianças autistas e não autista. Estes déficits funcionais eram previstos, na medida em que, independentemente do nível de funcionamento cognitivo, crianças autistas têm inabilidade de se relacionar com os outros e este déficit social afeta o desempenho cotidiano e adaptável dessas crianças ${ }^{12}$. Os resultados da Vineland no domínio social também mostram que crianças autistas apre sentam déficits sociais e conseqüentemente dificuldades na adaptação às exigências complexas das interações sociais, em que os mais baixos índices de socialização diferem crianças autistas de não autistas ${ }^{13}$.

Os resultados obtidos nos domínios verbais, de atividade de vida cotidiana e social permitiram obter o escore total da Vineland, ou seja, o nível de comportamento adaptativo da criança. Como esperado para uma amostra de crianças com autismo comparadas com crianças normais, os domínios da Vineland foram considerados abaixo dos níveis de comportamentos adaptáveis do grupo controle. Portanto, os resultados sugerem que as crianças autistas, quando avaliadas em termos de funcionalidade, apresentam índices de comportamento adaptativo comprometido quando comparadas com crianças normais. Isso permitiria pensar que essas crianças, ao apresentarem índices de adaptação comprometidos, teriam conseqüentemente pior QV, considerando que os déficits funcionais equivalem-se ao comprometimento das condições de bem-estar.

Porém, não podemos tomar por referencial apenas suas incapacidades ou déficits, para decidirmos se há ou não melhor QV. Pois, ao considerarmos a criança apenas sob a ótica da funcionalidade, deixa- mos em segundo plano algo bem mais relevante, que é o sujeito enquanto ser subjetivo, capaz de se expressar seus sentimentos e emoções. Por outro lado, o que qualifica o desempenho é o que o indivíduo faz no seu ambiente atual e este inclui o seu contexto social; também pode ser entendido como "a experiência vivida" das pessoas no contexto real em que vivem $^{14}$.

Entendemos que a "experiência vivida da criança" é um importante pressuposto do desempenho; porém, é também um importante referencial da QV subjetiva. Portanto, não se pode supor que desempenho abaixo dos parâmetros da normalidade seja sinônimo de baixa QV, na medida em que representa apenas um componente do estado de saúde da criança autista e não a sua globalidade. Assim, de acordo com os resultados obtidos em nosso estudo através da AUQEI, os índices gerais de QV foram iguais em ambos os grupos, indicando QV positiva. Esses resultados foram consistentes com estudos de QV realizados com amostragem infantil ${ }^{15,16}$.

Nos escores parciais da AUQEI, verificamos que o $g$ rupo re fe rente a funções não apresentou diferença significativa na percepção de crianças autistas quando comparadas com crianças normais: informações relacionadas à capacidade funcional não estariam completas se não levassem em consideração o grau de satisfação do sujeito ${ }^{17}$. A pontuação média para o $\mathrm{g}$ rupo re fe rente à família também não difere significativamente em relação ao grupo controle, possibilitando concluir que a família foi percebida como fonte de bem-estar para ambos os grupos. Portanto, corresponde uma instituição social significativa onde se inserea criança e suas interações ${ }^{18}$, com potencial de influência no processo de bem-estar subjetivo da criança autista. Igualmente, os índices que caracterizam lazer, não permitiram observar diferença estatisticamente significativa entre os dois grupos, conforme índices encontrados em outros estudos ${ }^{5,17}$; porém, no que se referem à autonomia, crianças autistas apresentaram índices mais elevados em relação ao grupo controle.

Os índices normais obtidos pelas crianças autistas no escore total da AUQEI, e nos subdomínios referentes a funções, família e férias e o índice mais elevado no que se refere à autonomia, se justificam na visão de que essas crianças apresentam a teoria da mente alterada ${ }^{19}$. Apresentam déficits na capacidade de levar em consideração seu próprio estado mental, bem como os dos outros. Esse déficit ocasionaria dificuldade em se colocar "no lugar das outras pessoas" 
ou em reconhecer o que a outra pessoa pode pensar ou sentir, o que ocasionaria menor crítica de si e do mundo. Isso permite que elas se auto-avaliem de maneira mais positiva e satisfatória do que uma criança de mesma idade, não autista, pois a última, por ter uma teoria da mente pre se nada, se vê e vê o outro a vendo, fazendo, portanto, avaliações e reavaliações de si mesma. $O$ resultado satisfaz a visão de que todo indivíduo, neste caso à criança autista, pode viver de forma saudável na sua condição de saúde ou doença, dependendo dos significados, atitudes e valores que atribuem às situações vivenciadas ${ }^{20}$.

Os resultados da AUQEI nos permitem questionar os processos que envolvem a habilitação das crianças autistas, enquanto influenciada pelo nível de desempenho funcional dessas crianças, incluindo o processo de educação, treinamento, motivação, características de personalidade, oportunidades sociais e condições médicas gerais. Os cuidados em relação à habilitação visam à cura e quando esta não é possível, busca-se o ajuste do indivíduo e mudança de comportamento ${ }^{14}$; isso nos levaria a refletir sobre o determinante papel que a percepção de vida da criança autista ocuparia na visão das estratégias de intervenções enquanto satisfatórias e adequadas aos seus intere s s es ou não.

Os resultados deste estudo sugerem que, muito além das expectativas dos outros, e/ou das dificuldades funcionais, a criança autista em sua percepção de mundo, é feliz, independentemente de seus déficits funcionais. Para assisti-las melhor, basta ouvi-la um pouco mais, mais do que qualquer processo de habilitação. As informações obtidas poderão garantirIhes um olhar pessoal, não só em termos de suas patologias. Ouvi-la, no seu universo pessoal, poderá abranger uma perspectiva de atendimento integral em saúde e permitir avaliar os efeitos dos procedimentos terapêuticos. Deve-se transcender, portanto, as fronteiras disciplinares e conceituais sejam elas biológicas, psicológicas, sociais e culturais, para const ruir uma lógica interior às particularidades de cada sujeito. Os resultados obtidos permitiram ainda concluir que crianças autistas apresentam índices de desenvolvimento adaptativo inferior, quando compara- das com crianças normais; crianças autistas, em sua percepção pessoal, apresentam índices de QV iguais ao de crianças normais, o que confirma nossa hipótese inicial.

\section{REFERÊNCIAS}

1. American Psychiatric Association. Diagnostic and statistical manual of mental disorders. $4^{\text {th }}$ Ed. Washington DC: APA, 1994.

2. Klin A. Attributing social meaning to ambiguous visual stimuli in higher-functioning autism and Asperger syndrome: the social attribution task. J Autism Develop Disord 2000;41:831-846.

3. Whoqol Group. Measuring quality of life: the development of the World Health Organization Quality of Life Instrument (WHOQOL). World Health Organization, 1993. In Bowling A (ed). Measuring health: a review of quality of life measurement scales. Buckingham: Open University Press, 1997.

4. Magnificat S, Dazord A. Évaluation de la qualité de vie de l'enfant: validation d'un questionnaire, premiers résultats. Neuropsychiatr Enfance Adolesc 1997;45:106-114.

5. Assumpção FB Jr, Kuczynski E, Sprovieri MH, Aranha EM. Escala de avaliação de qualidade de vida. Arq Neuropsiquiatr 2000;58:119-127.

6. Ballabriga MCJ, Escude RMC, Llaberia ED. Escala d`avaluación dels t rests autistes (ATA): validez de una escala para el examen de las conductas autistas. Rev Psiquiatr Infant Juv 1984;4:254-264.

7. Assumpção FB Jr, Kuczynski E, Gabriel ME, Rocca CC. Escala de avaliação de traços autísticos (ATA): validade e confiabilidade de uma escala para a detecção de condutas autísticas. Arq Neuropsiquiatr 1999; 57:23-29.

8. Sparrow SS, Balla DA, Ciccett DV. Vineland adaptive behavior scales. Circle Pines: American Guidance Service, 1984.

9. Magnificat S. Quality of life of children and adolescents after kidney or lives transplantation: child, parents and caregivers point of view. Pediatr Transplant 2003;7:228-235.

10. Szatmari P, A rdher L, Fisman S, Streiner DL, Wilson F. Asperger's syn$\mathrm{d}$ rome and autism: differences in behavior, cognition, and adaptive functioning. Am Acad Child Adolesc Psychiatry 1995;34:1662-1671.

11. Liss M, Harel B, Fein E, et al. Predictors and correlates of adaptive functioning in children with developmental disorders. J Autism Develop Disord 2001;31:219-230.

12. Carter AC, Volkmar FR, Pardal SS, et al. The adaptable scales of the behavior of Vineland: supplemental norms for individuals with autism. J Autism Develop Disord 1998;28:55-59.

13. Volkmar FR, Cohen D J. Co-morbid association of autism and schizophrenia. Am J Psychiatry 1991;48:1705-1707.

14. World Health Organization. International classification of functioning disability and health (ICF). Geneva: WHO, 2003.

15. Shear J. Aspects of the quality of life of children with a disability who a re in inclusive educational settings. Internat J Practical Appr Disab 2001;25:40-41.

16. Landolt MA, Nuoffer MJ, Steinmann B, Superti-Furga M. Quality of life and psychologic adjustment in children and adolescents with early treated phenylketonuria can be normal. J Pediatrics 2002;140:516-521.

17. Barreire SG, Oliveira OA, Kazama W, Kimura M, Santos A. Qualidade de vida de crianças ostomizadas na ótica das crianças e das mães. J Pediatria 2003;79:55-62.

18. Sprovieri MHS, Assumpção FB Jr. Dinâmica familiar de crianças autistas. Arq Neuropsiquiatri 2001; 59: 230-237.

19. Baron-Cohen S, Leslie AE, Frith D. Does the autistic child have a "theory of mind? Cognition 1985;21:37-46.

20. Anders JC. O transplante de medula óssea e suas repercussões na qualidade de vida de crianças e adolescentes que o vivenciaram. Tese. Ribeirão Preto, 2004. 